\title{
Tratamento de sementes com abamectina e Paecilomyces lilacinus no manejo de Heterodera glycines na cultura da soja
}

\author{
Janaína Alves de Almeidaㄹ ; Jean Cramenak de Souza ${ }^{1}$; Fernando Godinho de Araújo ${ }^{1 *}$
}

\author{
1Programa de Pós-Graduação em Proteção de Plantas, Instituto Federal Goiano - Campus Urutaí, GO, Brasil. *Autor para correspondência: \\ godinhoaraujo@hotmail.com. \\ 2Instituto Federal Goiano - Campus Urutaí, GO, Brasil.
}

\section{N F O A R T I G O}

\section{Histórico do artigo}

Recebido: 04 abril 2015

Aceito:21 maio 2015

\section{Palavras chaves:}

Controle biológico

Controle químico

Glycine max

Nematicidas

Nematoide de cisto da soja

\begin{abstract}
A B S T R A C T
Soybean is the most important oilseed crop worldwide, with Brazil the second largest producer. To maintain satisfactory levels of productivity efficient management is needed from several phytosanitary problems affecting the culture, including those caused by nematodes. Identify new management alternatives these nematodes it is necessary for maintaining low population levels in areas with the presence of these parasites. Thus, this study aimed to evaluate the efficiency of seed treatment with abamectin alone and associated with the fungus Paecilomyces lilacinus the management of Heterodera glycines in soybean. The test was conducted in a completely randomized design in a factorial 4 (abamectin doses) x 2 (with and without the addition of $P$. lilacinus), with 5 repetitions, using the soybean cultivar BRS Valiosa RR, susceptible to the nematode, in environment protected. Doses of abamectin employed were 0,50,100 and $200 \mathrm{~g}$ a.i. $100 \mathrm{~kg}$ seed -1 and P. lilacinus, 600 g c.p. $100 \mathrm{~kg}$ seed-1 (7,5x109 CFU / g PC). At 30 days after inoculation were analyzed fresh weight of the roots, the number of females per root system, females per gram and root nematode eggs per female. The fresh weight of roots and the number of females per root system were higher than in treatments without the addition of the fungus $P$. lilacinos. The treatment using the fungus $P$. lilacinus had a greater number of females per root gram as compared to treatment without the use of the fungus. The number of females per root system and eggs by females reduced due to the different doses of abamectin used. Abamectin alone and associated with the fungus P. lilacinus has potential use in the management of H. glycines in soybean.
\end{abstract}

\section{Introdução}

A soja (Glycine max (L.) Merril) é a mais importante oleaginosa cultivada mundialmente (Matos, 1987), sendo o Brasil o segundo maior produtor dos últimos cinco anos (USDA, 2015). O complexo soja colheu no Brasil, na safra 2014/15, cerca de 95.070,2 milhões de toneladas do grão, em uma área plantada de aproximadamente $31.573,0$ milhões de hectares, com uma produtividade média de 3.011 kg.ha-1 (Conab, 2015). Entretanto, para atingir índices consideráveis de produtividade é necessário controlar uma série de problemas fitossanitários que afetam a cultura, tendo destaque entre esses problemas, os fitonematoides.

São mais de cem espécies de nematoides abrangendo quase cinquenta gêneros associados à cultura da soja (Ferraz, 2001). No Brasil, Meloidogyne javanica e Meloidogyne incognita, Heterodera glycines, Pratylenchus brachyurus e Rotylenchulus reniformis são as espécies de maior importância e interesse econômico, devido aos danos provocados por elas, tais como a redução do crescimento da planta (dano direto) e, como porta de entrada para outros patógenos de solo (dano indireto) (Ferraz, 2001).

O H. glycines, conhecido como nematoide de cisto da soja, foi detectado pela primeira vez no Brasil na safra de 1991/92 (Lima et al., 1992; Lordello et al., 1992; Monteiro \& Morais, 1992) e desde então vem afetando negativamente o cultivo da soja no país. Nos Estados Unidos, nos anos de 2003 a 2005, foram registradas perdas de produtividade respectivamente de 2,9, 3,47 e 1,93 milhões de toneladas, provocadas pelo nematoide, fato esse que chama atenção para a sua severidade (Wrather \& Koenning, 2006). Dhingra et al. (2009) alertaram que as perdas de produtividade de grãos podem atingir $90 \%$, em combinação com o grau de infestação e raça do nematoide, fertilidade do solo e suscetibilidade da cultivar. 
Em áreas com a presença do $H$. glycines as três estratégias de manejo se baseiam na rotação de culturas, manejo do solo e uso de cultivares resistentes (Embrapa, 2015). Para Rigss \& Schmitt (1993) a rotação de culturas é eficiente no controle de $H$. glycines pelo número limitado de hospedeiros, sendo as culturas do milho, sorgo, trigo, cana de açúcar, algodão e girassol as mais empregadas (Yorinori, 2000). O uso de cultivares resistentes é o método mais empregado no mundo para o controle do NCS devido à eficiência e viabilidade econômica (Niblack et al., 2002). Porém, Riggs \& Schmitt (1993) advertem que o uso exclusivo dessa estratégia de manejo pode provocar pressão de seleção de raças e alterar a frequência genética do nematoide, visto que esse patógeno possui grande variabilidade.

Outras medidas como o controle químico (Pedrozo et al., 1999) e o uso de agentes de biocontrole (Timper \& Riggs, 1998; Hewlett et al., 2007) também tem sido utilizadas para tentar minimizar as perdas provocadas pelo nematoide, sendo o tratamento de sementes a forma mais economicamente viável de aplicação dessas estratégias. 0 tratamento de sementes tem o objetivo de suprimir o nematoide durante a fase inicial de desenvolvimento da cultura, proporcionando um adequado desenvolvimento do sistema radicular (Araujo, 2013). Produtos com ação nematicida à base de avermectina (abamectina) e metilcarbamato de oxima (tiodicarbe) são utilizados na cultura da soja via tratamento de semente (Monfort et al., 2006; Cabrera et al., 2009; Kubo et al., 2012).

Várias pesquisas com tratamento de sementes podem ser encontradas e trazem respostas satisfatórias dessa medida de controle para vários nematoides. Porém, para o $H$. glycines esses estudos ainda são escassos. Trabalhos como o Steffen et al. (2011) mostram a eficiência dos tratamentos de sementes com abamectina reduzindo o número J2 e J4 de Meloidogyne graminicola penetrados nas raízes do arroz irrigado e o de Bortolini et al. (2013) também comprova a eficiência de abamectina, aplicada em sementes de soja, reduzindo o nível populacional de Pratylenchus brachyurus.

Também neste contexto, vários gêneros de fungos podem ser utilizados no controle biológico e são capazes de atuar reduzindo a multiplicação de nematoides, tanto por predação quanto por antagonismo (Siddiqui \& Mahmood, 1996). O Paecilomyces lilacinus é um fungo de solo, que tem se mostrado promissor no biocontrole de espécies de Meloidogyne visto que, é um parasita facultativo de ovos e fêmeas desse nematoide (Kerry, 1990) e, oportunista com pouca especificidade de hospedeiro, sendo que sua eficácia varia em meio aos diferentes isolados (Goettel et al., 2001). As vantagens da utilização de fungos nematófagos (ovicidas ou oportunistas) para Atkins et al. (2003) é devido a capacidade saprofítica e fácil crescimento in vitro. Jacobs et al. (2003) acrescenta que este fungo é bastante competitivo em campo, adapta-se a uma ampla faixa de pH do solo e cresce em grande variedade de substrato.

Cadioli et al. (2009) avaliando o efeito de isolados de Paecilomyces lilacinus no desenvolvimento de cafezais e na população de $M$. paranaensis observaram que todos os isolados testados reduziram a população de J2 no solo. Santiago et al. (2006) verificaram que doze isolados de $P$. lilacinus conseguiram controlar M. paranaensis, reduzindo sua população em tomateiros.

Assim, o presente estudo teve como objetivo avaliar a eficiência do tratamento de sementes com abamectina isolada e associada com o fungo Paecilomyces lilacinus no manejo de Heterodera glycines na cultura da soja.

\section{Material e métodos}

0 experimento foi conduzido em ambiente protegido no Instituto Federal Goiano - Câmpus Urutaí, no município de Urutaí - GO. 0 delineamento experimental adotado foi inteiramente casualizado (DIC), sob esquema fatorial 4 (doses de abamectina) x 2 (com e sem a adição de P. lilacinus), com 5 repetições. 0 inóculo do nematoide cisto da soja (NCS) foi obtido de uma área naturalmente infestada do município de Ipameri - GO e multiplicados em plantas de soja suscetível (BRS Valiosa) em ambiente protegido.

Para implantação do ensaio, sementes de soja da cultivar BRS Valiosa RR, suscetível ao nematoide, foram previamente tratadas com doses de 50, 100, e $200 \mathrm{~g}$ de abamectina. $100 \mathrm{~kg}$ sementes $^{-1}$ e com P. lilacinus, 600 g p.c. 100 $\mathrm{kg}$ sementes $^{-1}\left(7,5 \times 10^{9} \mathrm{UFC} / \mathrm{g}\right.$ de PC). As sementes foram semeadas em vasos de cerâmica, com capacidade de 1,5 L, preenchidos por uma mistura de solo + areia, na proporção de 1:1, previamente esterilizado com fosfina. Semeou-se quatro sementes por vasos e posteriormente realizou-se o desbaste mantendo duas plântulas por vaso. A inoculação ocorreu quinze dias após a semeadura (DAS), adicionando-se uma suspenção com 4.000 ovos + J2 de H. glycines por vaso.

Aos trinta dias após a inoculação avaliou-se a massa fresca da raiz, o número de fêmeas por grama de raiz e o número de ovos por fêmea. Para extração das fêmeas as raízes de soja foram lavadas em água corrente sob conjunto de peneiras de 20 e 60 Mesh, seguindo a metodologia descrita por Tihohod (2000). Em seguida, o material retido na peneira de 20 Mesh foi descartado e o que ficou retido na peneira de 60 Mesh foi coletado e filtrado em papel filtro sobre uma calha telada (Andrade et al., 1995). Posteriormente, o material filtrado foi levado a microscópio estetoscópio (aumento de 15 x) para quantificação de fêmeas de $H$. glycines.

Para quantificação do número de ovos por fêmea, dez espécimes de cada repetição dos tratamentos foram separadas, aleatoriamente, e tiveram seu corpo rompido, sob jogo de peneiras de 100 sob 400 Mesh, com auxílio de um bastão. 0 material retido na peneira de 400 Mesh foi recolhido em béquer e o número de ovos quantificados em microscópio óptico (aumento de 50x) com o auxílio de uma câmara de Peters.

Os dados obtidos foram submetidos a análise de variância e as médias comparadas pelo teste de Tukey a 5\% de probabilidade. Todos os dados foram transformados em raiz quadrada de $\mathrm{x}+1$ e o software utilizado foi o SISVAR versão 5.4 (Ferreira, 2010).

\section{Resultados e discussão}

0 fungo $P$. lilacinus influenciou significativamente a massa fresca de raiz e, as quantidades de fêmeas por sistema radicular e por grama de raiz (Tabela 1). No entanto, não se observou diferença na quantidade de ovos produzidos por fêmeas (Tabela 1).

Tabela 1. Massa fresca de raiz, fêmeas por sistema radicular, fêmeas por grama de raiz e de ovos por fêmeas de H. glycines na cultivar de soja BRS Valiosa RR oriunda de sementes tratadas com diferentes doses de abamectina e com o fungo P. lilacinus, Urutaí - GO.

\begin{tabular}{|c|c|c|c|c|c|c|c|c|}
\hline \multirow{2}{*}{$\begin{array}{l}\text { Dose de } \\
\text { Abamec } \\
\text { tina }\end{array}$} & \multicolumn{2}{|c|}{$\begin{array}{c}\text { Massa } \\
\text { Fresca de } \\
\text { Raiz (g) }\end{array}$} & \multicolumn{2}{|c|}{$\begin{array}{l}\text { Fêmeas no } \\
\text { sistema } \\
\text { radicular }\end{array}$} & \multicolumn{2}{|c|}{$\begin{array}{c}\text { Fêmeas GR } \\
\text { Raiz }\end{array}$} & \multicolumn{2}{|c|}{$\begin{array}{c}\text { Ovos/Fême } \\
\text { a }\end{array}$} \\
\hline & $\begin{array}{c}\mathrm{S} / P . \\
\text { lilaci } \\
\text { nus }\end{array}$ & $\begin{array}{c}\mathrm{C} / P . \\
\text { lilaci } \\
\text { nus }\end{array}$ & $\begin{array}{l}\mathrm{S} / P . \\
\text { lilaci } \\
\text { nus }\end{array}$ & $\begin{array}{c}\mathrm{C} / P . \\
\text { lilaci } \\
\text { nus }\end{array}$ & $\begin{array}{c}\mathrm{S} / P . \\
\text { lilaci } \\
\text { nus }\end{array}$ & $\begin{array}{c}\mathrm{C} / P . \\
\text { lilacin } \\
\text { us }\end{array}$ & $\begin{array}{l}\text { S/P. } \\
\text { lilaci } \\
\text { nus }\end{array}$ & $\begin{array}{l}\mathrm{C} / P . \\
\text { lilaci } \\
\text { nus }\end{array}$ \\
\hline 0 & 1,75 & 0,33 & 49 & 33,6 & $\begin{array}{c}28,7 \\
5\end{array}$ & $\begin{array}{c}174,1 \\
1\end{array}$ & $\begin{array}{l}79,6 \\
3 \\
50,9\end{array}$ & $\begin{array}{l}74,2 \\
2 \\
76,3\end{array}$ \\
\hline 50 & 1,8 & 0,28 & 27 & 24,6 & $\begin{array}{l}14,9 \\
14,0\end{array}$ & 97,11 & 2 & $\begin{array}{l}6 \\
56,2\end{array}$ \\
\hline 100 & 2,04 & 0,38 & $\begin{array}{c}30 \\
31,7\end{array}$ & 16,6 & $\begin{array}{c}6 \\
13,5\end{array}$ & $\begin{array}{l}53,74 \\
117,8\end{array}$ & $\begin{array}{l}33,5 \\
66,5\end{array}$ & $\begin{array}{l}5 \\
56,7\end{array}$ \\
\hline & 28 & & 5 & 15 & 7 & 3 & 5 & 7 \\
\hline Média & $\begin{array}{c}1,57 \\
\mathrm{a}\end{array}$ & $\begin{array}{c}0,22 \\
\mathrm{~b}\end{array}$ & $\begin{array}{c}27,55 \\
\text { a }\end{array}$ & $\begin{array}{c}17,96 \\
\text { b }\end{array}$ & $\begin{array}{c}14,26 \\
\text { b }\end{array}$ & $\begin{array}{c}88,56 \\
a\end{array}$ & $\begin{array}{l}46,1 \\
2 \\
\end{array}$ & $\begin{array}{l}52,7 \\
2\end{array}$ \\
\hline CV (\%) & & & & & & 96 & & 1,79 \\
\hline
\end{tabular}


A utilização do fungo via tratamento de sementes reduziu a massa fresca de raiz. Tal resultado pode ter sido obtido em função da dosagem do fungo empregada no tratamento de sementes. Altas doses podem influenciar negativamente o desenvolvimento radicular da cultura. Cadioli et al. (2009) avaliando o efeito de isolados de $P$. lilacinus, sobre população de $M$. paranaenses, no desenvolvimento de cafezais observaram que todos os isolados do fungo, utilizados no experimento, também reduziram o peso fresco do sistema radicular.

Com relação ao número de fêmeas presentes no sistema radicular, observou-se redução em função da utilização do fungo P. lilacinus, (Tabela 1). Esse resultado se assemelha com o encontrado por Freitas et al. (1999), que para controlar $M$. javanica em mudas de tomateiro utilizaram substrato infestado com P. lilacinus e observaram redução do número de galhas por grama de raiz em $61,1 \%$ e por sistema radicular em 55,8 \%. Já o número de fêmeas por grama de raiz aumentou quando o fungo foi utilizado (Tabela 1). Esse resultado ocorreu em função da redução da massa fresca dos sistemas radiculares da soja tratada com $P$. lilacinus que consequentemente aumenta a proporção de fêmeas por grama de raiz.

No que diz à respeito das diferentes doses de abamectina, essas não influenciaram a massa fresca das raízes e o número de fêmeas por grama de raiz. No entanto, foram observadas diferenças significativas no número de fêmeas por sistema radicular e no número de ovos por elas produzidos (Figura 1). Ambas variáveis tiveram comportamento quadrático, sendo a dose de $100 \mathrm{~g}$ de i.a. $100 \mathrm{~kg}$ sementes $^{-1} \mathrm{a}$ que mais reduziu o número de fêmeas por sistema radicular e ovos por fêmeas (Figura 1). Observou-se ainda que independente da dose empregada de abamectina diminuiu o número de fêmeas por sistema radicular e no número de ovos produzidos por elas (Figura 1).

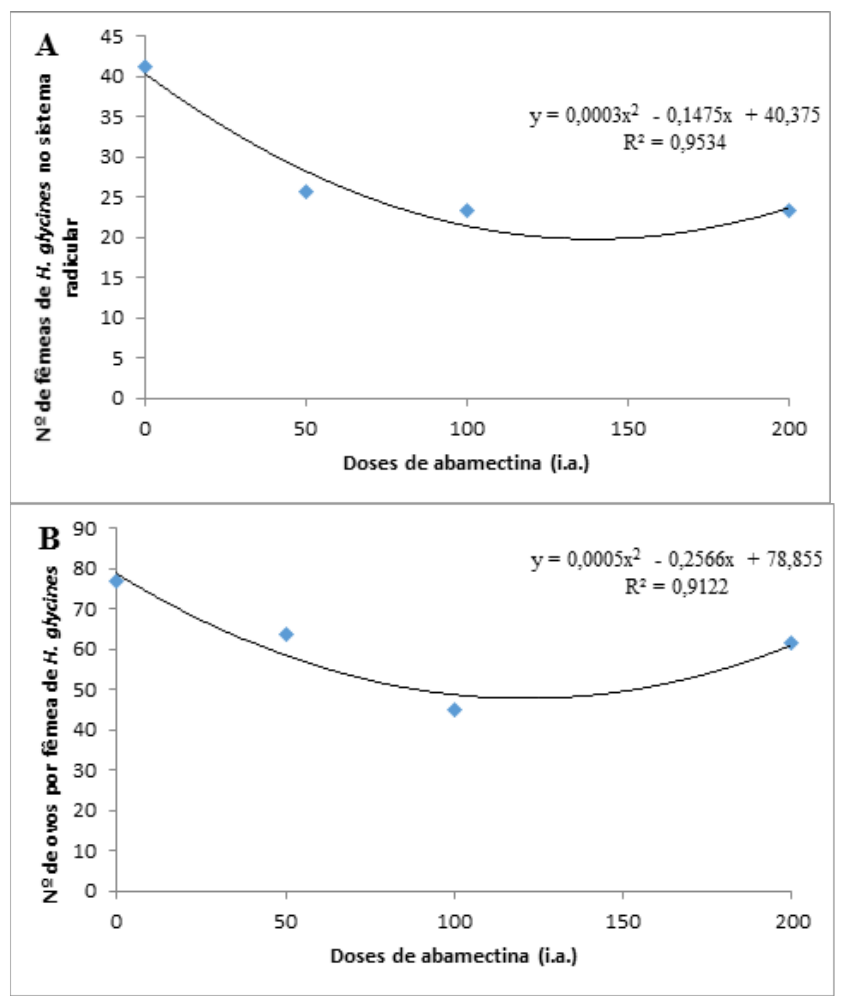

Figura 1.Tratamento de sementes com abamectina. A) Número de fêmeas por sistema radicular em função das doses de ingrediente ativo (i.a.) de abamectina por $100 \mathrm{~kg}$ de sementes. B) Número de ovos por fêmea em função das doses de i. a. de abamectina por $100 \mathrm{~kg}$ de sementes, Urutaí - GO.

Vale ressaltar que pesquisas com tratamento de sementes para controlar o nematoide $H$. glycines são escassos.
Porém, para o outros nematoides já existem vários estudos que apresentam resultados semelhantes aos encontrados. Steffen et al. (2011) também observaram redução do número de galhas, do nematoide M. graminicola, no sistema radicular das plantas de arroz que receberam tratamento químico de sementes, com abamectina e carbofuran, em relação as que não foram tratadas. A eficiência do tratamento de sementes de soja com abamectina também foi comprovada por Bortolini et al. (2013) que observaram redução populacional de $P$. brachyurus.

Pedrozo et al. (1999) verificaram, em casa de vegetação, que o tratamento com abamectina aplicado via foliar proporcionou melhor controle de $H$. glycines em soja que aldicarbe aplicado no solo. Faske \& Starr (2006) ao avaliarem a sensibilidade de $M$. incognita e $R$. reniformis a abamectina constatou a eficiência do produto, afirmando que concentrações de abamectina de 1,56 a 0,39 $\mu \mathrm{g} \cdot \mathrm{ml}^{-1}$ e 32,9 a 8,2 $\mu \mathrm{g} . \mathrm{ml}^{-1}$ foram letais aos nematoides $M$. incognita e $R$. reniformis, respectivamente, e reduziram a infectividade em raízes de tomate.

\section{Conclusão}

0 tratamento de sementes com abamectina mostrouse eficiente na redução de fêmeas de $H$. glycines por sistema radicular e de ovos por fêmeas em cultivar de soja suscetível ao nematoide.

A abamectina associada ao fungo $P$. lilacinus apresenta potencial de utilização no manejo de $H$. glycines na cultura da soja. No entanto, mais estudos são necessários para definição de doses que não afete o desenvolvimento do sistema radicular da cultura.

\section{Referências}

Atkins, S. D.; Hidalgo-Dias, L.; Kalisz, H.; Mauchline, T. H.; Hirsch, P. R.; Kerry, B. R. (2003). Development of a new management strategy for the control of root-knot nematodes (Meloidogyne ssp.) in organic vegetable production. Pest Management Science, 59, 183-189.

Araújo, F. G. (2013). Aspectos da biologia e manejo do nematoide de cisto da soja. (Tese de doutorado). Universidade Federal de Goiás, Brasil.

Bortolini, G. L.; Araújo, D. V.; Zavislak, F. D.; Romano Júnior, J.; Krause, W. (2014). Controle de Pratylenchus brachyurus via tratamento de semente de soja. Disponível em: <http://www.conhecer.org.br/enciclop/2013b/CIEN CIAS\%20AGRARIAS/CONTROLE\%20DE\%20Pratylenc hus.pdf> Acesso: 10/08/2014.

Cabrera, J. A.; Kiewnick, S.; Grimm, C.; Dabatat, A. A.; Sikora, R. A. (2009). Efficacy on abamectin seed treatment on Pratylenchus zeae, Meloidogyne incognita and Heterodera schachtii. Journal of Plant Diseases and Protection, 116 (3), 124-128.

Cadioli, M. C. et al. (2009). Efeito de isolados de Paecilomyces lilacinus no desenvolvimento de cafezais e na população de Meloidogyne paranaensis. Ciências Agrotec., 33 (3), 713-720.

Conab. Companhia Nacional de Abastecimento. (2015). Acompanhamento da safra brasileira. Disponível em: $<<$ http://www.conab.gov.br/OlalaCMS/uploads/arquiv os/15_05_12_08_59_36_boletim_graos_maio_2015.pdf > Acesso: 12/08/2015.

Embrapa, Empresa Brasileira de Pesquisa Agropecuária. (2015). Tecnologia de produção de soja região central do Brasil 2003. Disponível em: <<http://sistemasdeproducao.cnptia.embrapa.br/Fon tesHTML/Soja/SojaCentralBrasil2003/doenca.htm>> Acesso: 12/08/2015. 
Dhingra, O. D.; Mendonça, H. L.; Macedo, D. M. (2009). Doenças e seu controle. In: Sediyama, T. Tecnologias de produção e usos da soja. Londrina: Macenas, 133155.

Faske, T. R.; Starr, J. L. (2006). Sensitivity of Meloidogyne incognita and Rotylenchulus reniformis to abamectina. Journal of Nematology, 38 (2), 240-244.

Ferraz, L. C. B. (2001). As meloidoginoses da soja: passado, presente e futuro. In: Silva, J. F. V., Relações parasitohospedeiro nas meloidoginoses da soja (pp. 15-38). Londrina: Embrapa Soja / SBN.

Ferreira, D. F. (2010). Softwere Sisvar: versão 5.4.

Freitas, L. G.; Ferraz, S.; Almeida, A. M. S. (1999). Controle de Meloidogyne javanica em tomateiro pelas Mudas em Substrato Infestado com Paecilomyces lilacinus. Nematologia Brasileira, 23 (1), 65-73.

Gottel, M. S.; Hajek, E. A.; Siegel, J. P.; Evans, H. C. (2001). Safety of fungal biocontrole agentes. In: Butt, T. M.; Jackson, C.; Magan, N. Fungal as biocontrol agentes: problems, progress and potencial. Wallinford: Cabi, 347-376.

Hewlet, T. E.; Griswold, S. T.; Waters, J.; Smith, K. S. (2007). The potential for economic biological controlo f SCN using the bacterial parasite Pasteuria spp. 4th National Soybean Cyst Nematode Conference, p. 52.

Jacobs, H.; Gray, S. N.; Crump, D. H. (2003). Interactions between nematophagous fungi and consequences for their potential as biological agents for the control of potato cyst nematodes. Mycological Research, 107 (1), 47-56.

Kerry, B. R. (1990). An assessment of progress toward microbial controle of plant parasitic nematode. Journal of Nematology, 22 (45), 621-631.

Kubo, R. K.; Machado, A. C. Z.; Oliveira, C. M. G. (2012). Efeito do tratamento de sementes no controle de Rotylenchulus reniformis em dois cultivares de algodão. Arquivos Instituto Biológico, 59 (1/2), 239. 245.

Lima, R. D.; Ferraz, S.; Santos, J. M. (1992). Ocorrência de Heterodera sp., em soja no Triângulo Mineiro. Nematologia Brasileira, 16 (1/2), 101-102.

Lordello, A. I. L.; Lordellho, R. R. A.; Quaggio, J. A. (1992). Ocorrência do nematoide de cistos da soja (Heterodera glycines) no Brasil. Revista de Agricultura, 67 (3), 223-225.

Matos, M. P. (1987). Soja: a mais importante oleaginosa da agricultura moderna. São Paulo: Ícone editora Ltda, 1 73.

Monfort, W. S.; Kirkpatrick, T. L.; Long, D. L.; Rideout, S. (2006). Efficacy of a novel nematicidal seed treatment against Meloidogyne incognita on cotton. Journal of Nematology, 38 (2), 245-249.

Monteiro, A. R.; Morais, S. R. A. C. (1992). Ocorrência do nematoide de cisto da soja, Heterodera glycines Ichinohe, 1952, prejudicando a cultura no Mato Grosso do Sul. Nematologia Brasileira, 16 (1/2), 101.

Niblack, T. L.; Arelli, P. R.; Opperman, C. H.; Orf, J. H.; Schimitt, D. P.; Shennon, J. G.; Tylka, G. L. (2002). A revised classification scheme for genetically diverse populations of Heterodera glycines. Journal of Nematology, 34 (4), 279-288.

Pedroso, I. B.; Henning, A. A.; Homechin, M. (1999). Controle químico do nematoide de cisto da soja Heterodera glycines em casa de vegetação. Semina, 20 (1), 59-63.

Riggs, R. D.; Schmitt, D. P. (1993). Soybean cyst nematode. In: Sinclair, J. G.; Backman, P. A. Compendium of soybean disease. 3 ed., St. Paul, The American Phytopatological Society, 65-67.

Santiago, D. C. et al. (2006). Seleção de isolados de Paecilomyces lilacinus (Thom.) Samson para controle de Meloidogyne paraenses em tomateiro. Ciência Rural, 36 (4), 1055-1064.

Siddiqui, Z. A.; Mahmood, I. (1996). Biological control of plant parasitic nematodes by fungi. Bioresource Technology, 58 (3), 229-239.

Steffen, R. B.; Antoniolli, Z. I.; Steffen, G. P. K.; Jacques, R. J. S.; Echkardt, D. P. (2011). Efeito da abamectina e carbofuran no controle de danos causados por Meloidogyne graminicola em plantas de arroz irrigado. Revista da FZVA, 18 (2), 56-69.

Timper, P.; Riggs, R. D. (1998). Variation in efficacy of isolates of the fungus ARF against the soybean cyst nematode Heterodera glycines. Journal of Nematology, 30 (4), 461-467.

USDA, United States Department of Agricuture. (2015). Soja: análise da conjuntura agropecuária. Disponível em: << http://usdasearch.usda.gov/search?utf8=\%E2\%9C\% 93\&affiliate $=$ usda\&query $=$ conjuntura + da + soja $\& \mathrm{x}=0 \&$ $\mathrm{y}=0 \&$ commit=Search $>>$ Acessado: $18 / 08 / 2015$.

Wrather, J. A.; Koenning, S. R. (2006). Estimates od disease effects on soybean yields in the United States. Journal of Nematology, 38 (2), 173-180.

Yorinori, J. T. (2000). Riscos de surgimento de novas doenças na cultura da soja. In: Congresso de Tecnologia e competitividade da soja no mercado global. Fundação MT, Cuibá, 165-169 